\title{
A rigid Calabi-Yau manifold with Picard number two
}

Eberhard Freitag, 2015

\begin{abstract}
We study a projective Calabi-Yau threefold $\tilde{\mathcal{Y}}$ which has been constructed in [FS]. It is rigid $\left(h^{12}=0\right)$ and has Picard number $h^{11}=2$. We construct a pair of divisors $\mathcal{D}^{ \pm}$which give a basis of $\operatorname{Pic}(\tilde{\mathcal{Y}}) \otimes_{\mathbb{Z}} \mathbb{Q}$ and determine all intersection numbers $\mathcal{D}^{ \pm} \cdot \mathcal{D}^{ \pm} \cdot \mathcal{D}^{ \pm}$.
\end{abstract}

We want to thank Sergey V. Ketov, who motivated us to determine the intersection numbers which play a role for physical applications in string theory. We are grateful to S. Cynk who explained us several details about the resolution of nodes and we thank also R. Salvati Manni for widespread discussions and suggestions.

\section{Introduction}

Basic for our example is a certain complete intersection $\mathcal{X}$ of four quadrics introduced in the paper [GN] of van Geemen and Nygaard:

$$
\begin{aligned}
& Y_{0}^{2}=X_{0}^{2}+X_{1}^{2}+X_{2}^{2}+X_{3}^{2}, \\
& Y_{1}^{2}=X_{0}^{2}-X_{1}^{2}+X_{2}^{2}-X_{3}^{2}, \\
& Y_{2}^{2}=X_{0}^{2}+X_{1}^{2}-X_{2}^{2}-X_{3}^{2}, \\
& Y_{3}^{2}=X_{0}^{2}-X_{1}^{2}-X_{2}^{2}+X_{3}^{2} .
\end{aligned}
$$

The variety $\mathcal{X}$ has 96 isolated singularities which are ordinary double points (nodes).

In the paper $[\mathrm{CM}]$ it has been pointed out that the results of [GN] imply that $\mathcal{X}$ admits a resolution that is a (projective) Calabi-Yau threefold. The basic result - essentially due to van Geemen and Nygaard [GN] - is the following theorem.

Theorem. The Hodge numbers of a Calabi-Yau desingularization of $\mathcal{X}$ are

$$
h_{11}=32, \quad h_{12}=0 .
$$

Hence this Calabi-Yau manifold is rigid.

In the paper [FS], we constructed a certain group $G$ of order 16 of biholomorphic automorphisms of $\mathcal{X}$ that acts freely on $\mathcal{X}$. The basic thing is that there exists a projective small resolution $\tilde{\mathcal{X}}$ such that $G$ extends as group of biholomorphic mappings to $\tilde{\mathcal{X}}$. The quotient $\tilde{\mathcal{Y}}=\tilde{\mathcal{X}} / G$ then is a projective resolution of $\mathcal{Y}:=\mathcal{X} / G$ in form of a rigid Calabi-Yau manifold whose Picard number is two. 
The varieties $\mathcal{X}$ and $\mathcal{Y}$ are Siegel modular three folds. But for this paper the modular background is not necessary. Everything can be done by using the explicit equations.

We recall the definition of the group $G$. Let $P_{1}, \ldots, P_{8}$ be homogenous polynomials in $\mathbb{C}\left[Y_{0}, \ldots, Y_{3}, X_{0}, \ldots, X_{3}\right]$ of the same degree such that not all of them vanish. Then we can consider the rational map from $P^{7}$ into itself,

$$
\left(y_{0}, \ldots, y_{3}, x_{0}, \ldots, x_{3}\right) \longmapsto\left(P_{1}\left(y_{0}, \ldots, x_{3}\right), \ldots, P_{8}\left(y_{0}, \ldots, x_{3}\right)\right)
$$

We denote this map symbolically by

$$
\left(P_{1}, \ldots, P_{8}\right) \text {. }
$$

Theorem. The two transformations

$$
\begin{aligned}
& \left(-Y_{2}, \mathrm{i} Y_{3}, \mathrm{i} Y_{0}, Y_{1},-\mathrm{i} X_{3},-\mathrm{i} X_{2}, X_{1},-X_{0}\right), \\
& \left(\mathrm{i} Y_{1}, Y_{0},-\mathrm{i} Y_{3}, Y_{2}, X_{1}, \mathrm{i} X_{0}, X_{3},-\mathrm{i} X_{2}\right)
\end{aligned}
$$

define biholomorphic transformations of $\mathcal{X}$ onto itself. The group $G$ which is generated by the two biholomorphic transformations is isomorphic to $\mathbb{Z} / 4 \times \mathbb{Z} / 4$ and acts freely on $\mathcal{X}$. The quotient $\mathcal{Y}=\mathcal{X} / G$ has a resolution $\tilde{\mathcal{Y}}$ in the form of a (projective) rigid Calabi-Yau manifold $\left(h^{12}=0\right)$ with Euler number $e=4$ and Picard number $h^{11}=2$.

Proof. The proof is indicated in [FS]. We give some more details. The matrix group $\mathcal{G}$, generated by the two matrices

$$
\left(\begin{array}{cccccccc}
0 & 0 & -1 & 0 & 0 & 0 & 0 & 0 \\
0 & 0 & 0 & \mathrm{i} & 0 & 0 & 0 & 0 \\
\mathrm{i} & 0 & 0 & 0 & 0 & 0 & 0 & 0 \\
0 & 1 & 0 & 0 & 0 & 0 & 0 & 0 \\
0 & 0 & 0 & 0 & 0 & 0 & 0 & -\mathrm{i} \\
0 & 0 & 0 & 0 & 0 & 0 & -\mathrm{i} & 0 \\
0 & 0 & 0 & 0 & 0 & 1 & 0 & 0 \\
0 & 0 & 0 & 0 & -1 & 0 & 0 & 0
\end{array}\right) \quad\left(\begin{array}{cccccccc}
0 & \mathrm{i} & 0 & 0 & 0 & 0 & 0 & 0 \\
1 & 0 & 0 & 0 & 0 & 0 & 0 & 0 \\
0 & 0 & 0 & -\mathrm{i} & 0 & 0 & 0 & 0 \\
0 & 0 & 1 & 0 & 0 & 0 & 0 & 0 \\
0 & 0 & 0 & 0 & 0 & 1 & 0 & 0 \\
0 & 0 & 0 & 0 & \mathrm{i} & 0 & 0 & 0 \\
0 & 0 & 0 & 0 & 0 & 0 & 0 & 1 \\
0 & 0 & 0 & 0 & 0 & 0 & -\mathrm{i} & 0
\end{array}\right)
$$

has order 64. Its center $\mathcal{C}$ is the group of order 4 , generated by the matrix $\mathrm{i} E$ (where $E$ denotes the unit matrix). There is a natural homomorphism $\mathcal{G} \rightarrow G$ which gives the identification $\mathcal{G} / \mathcal{Z} \cong G$. (As usual, $n \times n$-matrices act from the left on $\mathbb{C}^{n}$ : the elements of $\mathbb{C}^{n}$ are written as columns and then matrices act by matrix multiplication from the left).

Next we prove that $G$ acts fixed point free on $\mathcal{X}$. It is enough to do this for elements of order two in $G$. There are three of them, namely the two squares of the two generators and the product of the two squares. All three are diagonal matrices with two different entries. It is very easy to check that they have no fixed point in $\mathcal{X}$. 


\section{Intersection numbers between basic divisors}

In the paper [FS], 188 two-dimensional subvarieties of $\mathcal{X}$ have been defined. We use here two of them. They are components of the hyperplane section $X_{0}+\cdots+X_{3}=0$. One of them is cut out by the equations

$$
D^{+}: \quad X_{0}+X_{1}+X_{2}+X_{3}=Y_{1}\left(X_{1}+X_{3}\right)+(\sqrt{2} / 2) Y_{2} Y_{3}=0
$$

The vanishing ideal of this variety is the radical of the ideal generated by the defining ideals of $\mathcal{X}$ and $D^{+}$. It can be computed as

$$
\begin{aligned}
& Y_{0}^{2}-2 X_{1}^{2}-2 X_{1} X_{2}-2 X_{1} X_{3}-2 X_{2}^{2}-2 X_{2} X_{3}-2 X_{3}^{2} \\
& Y_{1}^{2}-2 X_{1} X_{2}-2 X_{1} X_{3}-2 X_{2}^{2}-2 X_{2} X_{3} \\
& Y_{1} Y_{2}+\sqrt{2} Y_{3} X_{1}+\sqrt{2} Y_{3} X_{2} \\
& Y_{1} Y_{3}+\sqrt{2} Y_{2} X_{2}+\sqrt{2} Y_{2} X_{3} \\
& Y_{1} X_{1}+Y_{1} X_{3}+1 / 2 \sqrt{2} Y_{2} Y_{3} \\
& Y_{2}^{2}-2 X_{1}^{2}-2 X_{1} X_{2}-2 X_{1} X_{3}-2 X_{2} X_{3} \\
& Y_{3}^{2}-2 X_{1} X_{2}-2 X_{1} X_{3}-2 X_{2} X_{3}-2 X_{3}^{2} \\
& X_{0}+X_{1}+X_{2}+X_{3}
\end{aligned}
$$

Replacing $\sqrt{2}$ by $-\sqrt{2}$ we get a complementary ideal

$$
D^{-}: \quad X_{0}+X_{1}+X_{2}+X_{3}=Y_{1}\left(X_{1}+X_{3}\right)-(\sqrt{2} / 2) Y_{2} Y_{3}=0
$$

In the following we use the notation $D^{ \pm}$for the corresponding irreducible divisors. We recall some basics about of divisors and their intersection numbers.

Let $X$ be a normal irreducible algebraic variety of dimension $n$ over $\mathbb{C}$. A divisor on $X$ is a formal sum of irreducible closed subvarieties of codimension one. Let $Y \subset X$ be a subvariety of everywhere codimension 1. The associated divisor is the sum of all irreducible components (with multiplicities 1). To every rational function its principal divisor can be associated. Two divisors are called equivalent if their difference is principal. Since the singular locus of $X$ has codimension $\geq 2$, the divisors (divisor classes) on $X$ are in one-to-one correspondence with the divisors (divisor classes) on the regular locus. Let $\pi: \tilde{X} \rightarrow X$ be a small resolution. Small means that there exists a closed subvariety $T \subset \tilde{X}$ of codimension $\geq 2$ such that $\pi$ defines a biholomorphic map of $\tilde{X}-T$ onto the regular locus of $X$. The divisors (divisor classes) of $X$ are in one-to-one correspondence with those of $\tilde{X}$.

Now we assume that $X$ is projective and non-singular. Then the intersection number $D_{1} \cdots D_{n}$ of $n$ divisors $D_{1}, \ldots D_{n}$ can be defined. It is invariant under equivalence and it is $\mathbb{Z}$-multilinear. In the case that the divisors are effective 
and intersect only in finitely many points $P_{1}, \ldots, P_{m}$, the intersection number is the sum of the multiplicities of $P_{i}$ in the scheme theoretic intersection $D_{1} \cap$ $\ldots \cap D_{n}$. For details we refer to [Sh].

We consider a projective small resolution $\tilde{\mathcal{X}}$. (This is a Calabi-Yau manifold). We can consider divisors $D_{1}, D_{2}, D_{3}$ on $\mathcal{X}$ as divisors on $\tilde{\mathcal{X}}$ and then study intersection numbers of three divisors. To be precise, $D_{1} \cdot D_{2} \cdot D_{3}$ means the intersection number on $\tilde{\mathcal{X}}$. These numbers may depend on the choice of the resolution. But, if one of the three divisors is equivalent to a divisor which avoids all nodes, then the intersection is independent of the choice. For example, this is the case for hyper plane sections. They are all equivalent and there is one which avoids a finite number of given points.

We consider the divisors $D^{+}$and $D^{-}$on $\mathcal{X}$. Their sum $H$ is the divisor of a hyperplane section. We can consider the three divisors also as divisors on $\tilde{\mathcal{X}}$. In this section we want to compute the intersection numbers of three of the divisors $H, D^{+}, D^{-}$(on $\left.\tilde{\mathcal{X}}\right)$. As we explained, they to not depend on the choice of the small resolution.

1.1 Proposition. Let $H$ be a divisor on $\mathcal{X}$ that represents a hyperplane section, i.e. $H \sim\left(X_{0}\right)$. Then

$$
H \cdot H \cdot H=16 \text {. }
$$

Proof. The hyperplane sections $\left(X_{0}\right),\left(X_{1}\right),\left(X_{2}\right)$ have (on $\left.\mathcal{X}\right) 16$ intersection points,

$$
[ \pm 1, \pm \mathrm{i}, \pm \mathrm{i}, \pm 1,0,0,0,1] .
$$

None of them is a node. Their multiplicity is one.

1.2 Proposition. Let $H$ be a divisor on $\mathcal{X}$ that represents a hyperplane section, i.e. $H \sim\left(X_{0}\right)$. Then

$$
H \cdot H \cdot D^{+}=H \cdot H \cdot D^{-}=8 .
$$

Proof. There is a biholomorphic transformation of $\mathcal{X}$ that sends $Y_{2}$ to $-Y_{2}$ and preserves the remaining variables. The divisor class of $H$ remains fixed and $D^{+}$and $D^{-}$are interchanged. Hence $H \cdot H \cdot D^{+}=H \cdot H \cdot D^{-}$. Their sum is 16 by Proposition 1.1. (We should mention that a biholomorphic transformation usually does not extend to $\tilde{\mathcal{X}}$. But there is a second resolution $\overline{\mathcal{X}}$ such that it extends to a biholomorphic map $\tilde{X} \rightarrow \overline{\mathcal{X}}$. This shows that $H \cdot H \cdot D^{+}$, computed on $\tilde{\mathcal{X}}$, equals $H \cdot H \cdot D^{-}$, computed on $\overline{\mathcal{X}}$. But as we noted already these intersection numbers are independent of the choice of a small resolution.) 
1.3 Proposition. Let $H$ be a hyperplane section, i.e. $H \sim\left(X_{0}\right)$. Then

$$
H \cdot D^{+} \cdot D^{-}=20, \quad H \cdot D^{+} \cdot D^{+}=H \cdot D^{-} \cdot D^{-}=-12 .
$$

Proof. The divisors $\left(Y_{0}\right), D^{+}, D^{-}$have 12 intersection points, None of them is a node. The 4 points

$$
[0, \pm 2 \mathrm{i}, 0,0,-\mathrm{i},-1, \mathrm{i}, 1], \quad[0, \pm 2 \mathrm{i}, 0,0, \mathrm{i},-1,-\mathrm{i}, 1]
$$

have multiplicity 3 . The remaining 8

$$
\begin{aligned}
& {[0,0, \pm 2 \mathrm{i}, 0,-\mathrm{i}, \mathrm{i},-1,1], \quad[0,0, \pm 2 \mathrm{i}, 0, \mathrm{i},-\mathrm{i},-1,1]} \\
& {[0,0,0, \pm 2,-1, \mathrm{i},-\mathrm{i}, 1], \quad[0,0,0, \pm 2,-1,-\mathrm{i}, \mathrm{i}, 1]}
\end{aligned}
$$

have multiplicity 1 . This gives the intersection number $4 \cdot 3+8=20$. The rest follows with the help of Proposition 1.2.

1.4 Proposition. We have

$$
\begin{aligned}
& D^{+} \cdot D^{+} \cdot D^{+}=D^{-} \cdot D^{-} \cdot D^{-}=-22 \\
& D^{+} \cdot D^{+} \cdot D^{-}=D^{+} \cdot D^{-} \cdot D^{-}=10 .
\end{aligned}
$$

Proof. We have

$$
D^{+} \cdot D^{+} \cdot D^{-}=D^{+} \cdot D^{-} \cdot D^{-} \text {. }
$$

The sum of both is 20 (Proposition 1.3). Hence we obtain the value 10 . We also have

$$
D^{+} \cdot D^{+} \cdot D^{+}=D^{+} \cdot D^{+} \cdot H-D^{+} \cdot D^{+} \cdot D^{-}=-22 \text {. }
$$

This completes the proof of Proposition 1.4.

\section{Intersection numbers between translates}

2.1 Proposition. We have

$$
H \cdot H \cdot g\left(D^{+}\right)=H \cdot H \cdot g\left(D^{-}\right)=8 \text {. }
$$

This follows from Proposition 1.2. 
2.2 Proposition. We have

$$
H \cdot g\left(D^{+}\right) \cdot g\left(D^{-}\right)=20, \quad H \cdot g\left(D^{+}\right) \cdot g\left(D^{+}\right)=H \cdot g\left(D^{-}\right) \cdot g\left(D^{-}\right)=-12 \text {. }
$$

This follows from Proposition 1.3.

2.3 Proposition. We have

$$
H \cdot g_{1}\left(D^{+}\right) \cdot g_{2}\left(D^{+}\right)=H \cdot g_{1}\left(D^{+}\right) \cdot g_{2}\left(D^{-}\right)=H \cdot g_{1}\left(D^{-}\right) \cdot g_{2}\left(D^{-}\right)=4
$$

for all different $g_{1} \neq g_{2}$ in $G$.

Proof. It is sufficient to treat the case where $g_{1}$ is the identity. Then $g=g_{2}$ is different from the identity. In this case the intersection of $Y_{0}=0, D^{+}$and $g\left(D^{+}\right)$consists of 4 points. They are members of the following list of 12 points.

$$
\begin{array}{lll}
{[0,0,0,-2,-1,-\mathrm{i}, \mathrm{i}],} & {[0,0,-2 \mathrm{i}, 0, \mathrm{i},-\mathrm{i},-1],} & {[0,0,-2 \mathrm{i}, 0,-\mathrm{i}, \mathrm{i},-1],} \\
{[0,0,2 \mathrm{i}, 0, \mathrm{i},-\mathrm{i},-1],} & {[0,2 \mathrm{i}, 0,0, \mathrm{i},-1,-\mathrm{i}],} & {[0,0,0,2,-1,-\mathrm{i}, \mathrm{i}],} \\
{[0,0,2 \mathrm{i}, 0,-\mathrm{i}, \mathrm{i},-1],} & {[0,-2 \mathrm{i}, 0,0,-\mathrm{i},-1, \mathrm{i}],} & {[0,0,0,2,-1, \mathrm{i},-\mathrm{i}],} \\
{[0,2 \mathrm{i}, 0,0,-\mathrm{i},-1, \mathrm{i}],} & {[0,0,0,-2,-1, \mathrm{i},-\mathrm{i}],} & {[0,-2 \mathrm{i}, 0,0, \mathrm{i},-1,-\mathrm{i}] .}
\end{array}
$$

None of them is a a node. They have multiplicity one. This shows $H \cdot g_{1}\left(D^{+}\right)$. $g_{2}\left(D^{+}\right)=4$. The rest follows from Proposition 2.1.

2.4 Proposition. For $g \in G$ we have

$$
\begin{aligned}
& g\left(D^{+}\right) \cdot g\left(D^{-}\right) \cdot g\left(D^{-}\right)=g\left(D^{+}\right) \cdot g\left(D^{+}\right) \cdot g\left(D^{-}\right)=10 \\
& g\left(D^{+}\right) \cdot g\left(D^{+}\right) \cdot g\left(D^{+}\right)=g\left(D^{-}\right) \cdot g\left(D^{-}\right) \cdot g\left(D^{-}\right)=-22 .
\end{aligned}
$$

This follows from Proposition 1.4.

2.5 Proposition. Let $g_{1}, g_{2}, g_{3}$ be three elements from $G$ which contain two different ones, $\#\left\{g_{1}, g_{2}, g_{3}\right\}=2$. Then we have

$$
g_{1}\left(D^{ \pm}\right) \cdot g_{2}\left(D^{ \pm}\right) \cdot g_{3}\left(D^{ \pm}\right)=10
$$

if the three factors $g_{i}\left(D^{ \pm}\right)$are pairwise different. For all other sign combinations we have

$$
g_{1}\left(D^{ \pm}\right) \cdot g_{2}\left(D^{ \pm}\right) \cdot g_{3}\left(D^{ \pm}\right)=-6
$$

Proof. Applying the transformation $Y_{2} \mapsto-Y_{2}$, we see $g_{1}\left(D^{+}\right) \cdot g_{2}\left(D^{+}\right)$. $g_{2}\left(D^{-}\right)=g_{1}\left(D^{-}\right) \cdot g_{2}\left(D^{+}\right) \cdot g_{2}\left(D^{-}\right)$. Their sum is 20 by Proposition 2.2. This gives that both sides are 10 . The proof of the rest is similar.

For the proofs we use computer calculations. For this one needs a base field. We take $K=\mathbb{Q}(\zeta)$ where $\zeta$ is an 8 th root of unity. The varieties $D^{ \pm}$ are irreducible over $K$. We think that they are irreducible over $\mathbb{C}$ but it is not necessary to know this. 
2.6 Proposition. Let $\left(g_{1}, g_{2}, g_{3}\right)$ be three pairwise different elements of $G$. Then

$$
g_{1}\left(D^{ \pm}\right) \cdot g_{2}\left(D^{ \pm}\right) \cdot g_{3}\left(D^{ \pm}\right)=2 .
$$

Proof. There have to be treated the following cases. Let $K$ be the subgroup of $G$ that is generated by $g_{1}^{-1} g_{2}$ and $g_{1}^{-1} g_{3}$.

1) The order of $K$ is 16 . There are $16 \cdot 96$ triples with this property. There are two subcases:

a) In $16 \cdot 48$ cases the intersection of $g_{1}\left(D^{ \pm}\right), g_{2}\left(D^{ \pm}\right), g_{3}\left(D^{ \pm}\right)$consists of two points which are not nodes and which have multiplicity one and which both are defined over $K$.

b) In $16 \cdot 48$ cases the intersection of $g_{1}\left(D^{ \pm}\right), g_{2}\left(D^{ \pm}\right), g_{3}\left(D^{ \pm}\right)$consists of two points which are not nodes and which have multiplicity one and which both are not defined over $K$.

2) The order of $K$ is 8 . There are two subcases:

a) In $16 \cdot 36$ cases there are two intersection points which are defined over $\mathbb{Q}(\mathrm{i})$. None of them is a node and the multiplicity is in each case 1.

b) In $16 \cdot 36$ cases there are 4 intersection points. They are all nodes. Here one has to take the intersection numbers in the small resolution $\tilde{\mathcal{X}}$. In two of the four one has no intersection point. In each of the other two there is one intersection point with multiplicity one. This case will be treated in detail in the following Section.

3) The order of $K$ is 4 . There are $16 \cdot 42$ cases. In all of them there are 2 intersection points which are defined over $\mathbb{Q}(\mathrm{i})$. They are not nodes and they have multiplicity 1.

In each of the cases only one sign combination, for example three plus signs, have to be treated. The other combinations then one can obtain with the help of Proposition 2.3. The only really difficult case is $2 \mathrm{~b}$ ). In all other cases we have intersection points only outside of the nodes and we can work with the variety $\mathcal{X}$ and have not to desingularize it. As in Sect. 2 , the intersection points can be computed in each case explicitly and after that one can compute their multiplicities. The case $2 \mathrm{~b}$ ) is more involved. We will study this case in the next section.

\section{Intersections in exceptional fibres}

In this section we explain the case $2 \mathrm{~b}$ ) in the proof of Proposition 2.6, the only case where nodes come into the game.

Before we start, we have to recall some details about the resolutions of a node. First, one can consider the blow up of a node. The exceptional fibre is 
biholomorphic equivalent to a $P^{1} \times P^{1}$. After a biholomorphic map has been chosen, we can talk about horizontal lines $P^{1} \times\left\{a_{2}\right\}$ and vertical lines $\left\{a_{1}\right\} \times P^{1}$. A different choice of the biholomorphic map can preserve "horizontal" and "vertical" or exchange them. One can contract one of the two types of lines to obtain a small resolution with exceptional fibre $P^{1}$. Hence there exist two essentially different small resolutions. The choice of the small resolution is called a ruling of the node. We recall the following fact. Let $Y \subset \mathcal{X}$ be a pure two dimensional subvariety which is smooth at a node. Then the blow-up of $Y$ gives one of the two small resolutions. The strict transform in the blow up of the node is a horizontal or vertical line.

The following Lemma has been communicated to us by S. Cynk.

3.1 Lemma. Assume that $a$ is a node in $\mathcal{X}$ and that $Y_{1}, Y_{2}$ are two smooth surfaces which contain a. In the case that the node $a$ is an (maybe embedded) component of the scheme theoretic intersection $Y_{1} \cap Y_{2}$, the corresponding lines in $P^{1} \times P^{1}$ must be parallel (including equal), i.e. both horizontal, or both vertical. In the other cases the two lines intersect properly (one horizontal, one vertical).

Additional Remark. In the case of parallel lines, the two lines are equal if and only if the tangent planes of $Y_{1}, Y_{2}$ at a are the same.

Assume that we have three surfaces $Y_{1}, Y_{2}, Y_{3}$ which have the node $a$ as isolated intersection point and which are smooth at $a$. We consider their strict transforms in the exceptional fibre $P^{1} \times P^{1}$ of the blow up of the node. These can be three parallel lines (equal lines are considered to be parallel) or two parallel lines and a further line intersecting them.

3.2 Definition. Let $Y_{1}, Y_{2}, Y_{3}$ be three surfaces in $\mathcal{X}$ which have the node a as isolated intersection point and which are smooth at a. They are called in good position (at a) if their strict transform in the blow up of the node consists of two different parallel lines and one which intersects them.

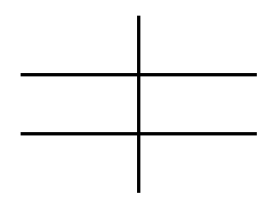

We contract this figure in horizontal and vertical direction.

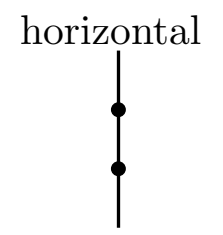

vertical

In the horizontal direction we see one line and two points on it. The intersection of these three is empty. In the vertical direction we see one line and one point on it. The intersection is one point. Hence we see the following result. 
3.3 Proposition. Assume that the node a is ruled (so horizontal and vertical is defined). Assume that we have two triples of surfaces $\left(D_{1}^{\prime}, D_{2}^{\prime}\right.$. $\left.D_{3}^{\prime}\right)$, $\left(D_{1}^{\prime \prime}, D_{2}^{\prime \prime}, D_{3}^{\prime \prime}\right)$ which both are in good position in the sense of Definition 3.2. Assume that their two diagrams are different (not parallel) in the sense of the following figure.
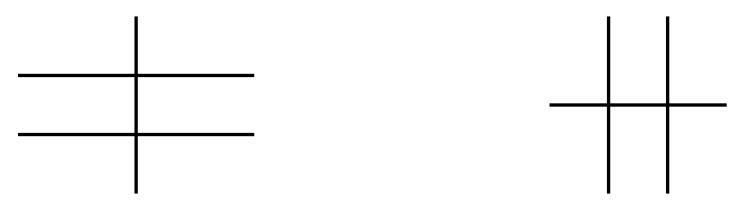

Let $\alpha^{\prime}$ be the number of intersection points of $D_{1}^{\prime}, D_{2}^{\prime}, D_{3}^{\prime}$ in the exceptional $P^{1}$ and similarly $\alpha^{\prime \prime}$. Then

$$
\alpha^{\prime}+\alpha^{\prime \prime}=1
$$

In particular, this sum is independent of the choice of the ruling.

We consider an example for Proposition 2.6, 2b):

$$
\begin{aligned}
& g_{1}:=\text { identity } \\
& g_{2}:=\left(-Y_{0}, Y_{1},-Y_{2}, Y_{3}, X_{0},-X_{1},-X_{2}, X_{3}\right), \\
& g_{3}:=\left(\mathrm{i} Y_{3},-\mathrm{i} Y_{2},-Y_{1},-Y_{0}, X_{2},-\mathrm{i} X_{3}, \mathrm{i} X_{0}, X_{1}\right) .
\end{aligned}
$$

Recall that $D^{+}$is a component of the hyperplane section $X_{0}+X_{1}+X_{2}+X_{3}=0$. The intersection of the three divisors

$$
g_{1}\left(D^{+}\right), \quad g_{2}\left(D^{+}\right), \quad g_{3}\left(D^{+}\right)
$$

considered in $\mathcal{X}$, consists of 4 nodes.

$$
\begin{aligned}
& a_{1}:=[\sqrt{2}, 0,0,-\sqrt{2} \mathrm{i}, 0,-1,1], \\
& a_{2}:=[\sqrt{2}, 0,0, \sqrt{2} \mathrm{i}, 0,-1,1], \\
& a_{3}:=[-\sqrt{2}, 0,0,-\sqrt{2}, 0,-1,1], \\
& a_{4}:=[-\sqrt{2}, 0,0, \sqrt{2}, 0,-1,1] .
\end{aligned}
$$

Unfortunately the three divisors are not in good position with respect to all nodes $a_{1}, \ldots, a_{4}$ in the sense of Definition 3.2. For example at the second node $a_{2}$ the following happens. The curves $g_{1}\left(D^{+}\right) \cap g_{3}\left(D^{+}\right)$and $g_{2}\left(D^{+}\right) \cap g_{3}\left(D^{+}\right)$ have $a_{2}$ as isolated fixed point. But the intersection of $g_{1}\left(D^{+}\right)$and $g_{3}\left(D^{+}\right)$in $\mathcal{X}$ is set theoretically an elliptic curve, cut out in $\mathcal{X}$ by the linear equations

$$
Y_{1}=Y_{2}=X_{0}+X_{3}=X_{1}+X_{2}
$$

But there are four embedded components in this curve, namely the 4 nodes. Hence the three lines in the exceptional $P^{1} \times P^{1}$ of the node are parallel. 
To remedy this situation, we replace the divisor $g_{1}\left(D^{+}\right)$by the other component $g_{1}\left(D^{-}\right)$. From Proposition 2.3 we see that

$$
g_{1}\left(D^{+}\right) \cdot g_{2}\left(D^{+}\right) \cdot g_{3}\left(D^{+}\right)+g_{1}\left(D^{-}\right) \cdot g_{2}\left(D^{+}\right) \cdot g_{3}\left(D^{+}\right)=4
$$

Hence we see

$$
g_{1}\left(D^{+}\right) \cdot g_{2}\left(D^{+}\right) \cdot g_{3}\left(D^{+}\right)=2 \Longleftrightarrow g_{1}\left(D^{-}\right) \cdot g_{2}\left(D^{+}\right) \cdot g_{3}\left(D^{+}\right)=2
$$

3.4 Remark. We use now the notation

$$
D_{1}=g_{1}\left(D^{-}\right), \quad D_{2}=g_{2}\left(D^{+}\right), \quad D_{3}=g_{3}\left(D^{+}\right)
$$

a) The intersection of $D_{1} \cap D_{2} \cap D_{3}$ consists of the same 4 nodes $a_{1}, \ldots, a_{4}$.

b) The three divisors $D_{1}, D_{2}, D_{3}$ are in good position at all 4 nodes.

We can use Lemma 3.1 to clarify the position of two of the $D_{i}$. The computation can be done with help of MAGMA which allows to compute the primary decomposition of an ideal.

We need now some information about the small desingularization $\tilde{\mathcal{X}}$. The group $G$ has the essential fact that it extends to $\tilde{\mathcal{X}}$. This means that it preserves the rulings of the nodes. We use this to compare the rulings of the nodes $a_{1}, a_{2}, a_{3}, a_{4}$. One checks

$$
g_{1}\left(a_{2}\right)=a_{1}, \quad g_{1}\left(a_{4}\right)=a_{3}
$$

We want to compare the number of intersection points of $D_{1}, D_{2}, D_{3}$ over the node $a_{1}$ with those over $a_{2}$. Instead of this we can compare

a) the number of intersection points of $D_{1}, D_{2}, D_{3}$ over the node $a_{1}$,

b) the number of intersection points of $g_{1}\left(D_{1}\right), g_{1}\left(D_{2}\right), g_{1}\left(D_{2}\right)$ over $a_{1}$.

One can compute the positions of the line diagrams in the sense of Proposition 3.3. It turns out that the two diagrams are different. So we can apply Proposition 3.3 to obtain the following result.

The divisors $D_{1}, D_{2}, D_{3}$ have an intersection point in the small resolution over $a_{1}$ or $a_{2}$ but not over both. The same argument works for the pair $a_{3}, a_{4}$. Hence we get

$$
D_{1} \cdot D_{2} \cdot D_{3}=2 \text {. }
$$

We settled an example for 2b). Each other case of Proposition 2.6. can be treated in the same manner. This completes the proof of Proposition 2.6, 2b). 


\section{Intersection numbers in the quotient}

We recall from the paper [FS].

4.1 Theorem. The orbit of the subvariety $D^{+}$(the same is true for $D^{-}$) under the group $G$ consists of 16 subvarieties of $\mathcal{X}$. They are non-singular. Each of the 96 nodes is contained in 4 of the 16 subvarieties. The blow up along the union of the 16 (considered as reduced subvariety) is a smooth projective variety $\tilde{\mathcal{X}}$ with a free $G$-action. The quotient $\tilde{\mathcal{X}} / G$ is a (projective) rigid CalabiYau manifold with $h^{11}=2(e=4)$.

From $[\mathrm{FS}]$ we know the following result.

4.2 Proposition. We denote by $\mathcal{D}^{ \pm}$the image of $D^{ \pm}$in $\tilde{\mathcal{X}} / G$. The two divisors give a $\mathbb{Q}$-basis of

$$
\operatorname{Pic}(\tilde{\mathcal{X}} / G) \otimes_{\mathbb{Z}} \mathbb{Q}
$$

We want to compute the intersection numbers $\mathcal{D}^{ \pm} \cdot \mathcal{D}^{ \pm} \cdot \mathcal{D}^{ \pm}$. These are essentially 4 cases, depending on the number of the plus (or minus) signs. Let $\pi: \mathcal{X} \rightarrow \mathcal{X} / G$ be the canonical projection. We use the formula

$$
16 \mathcal{D}^{ \pm} \cdot \mathcal{D}^{ \pm} \cdot \mathcal{D}^{ \pm}=\pi_{*}\left(\pi^{*} \mathcal{D}^{ \pm} \cdot \pi^{*} \mathcal{D}^{ \pm} \cdot \pi^{*} \mathcal{D}^{ \pm}\right)
$$

It implies

$$
16 \mathcal{D}^{ \pm} \cdot \mathcal{D}^{ \pm} \cdot \mathcal{D}^{ \pm}=\pi^{*} \mathcal{D}^{ \pm} \cdot \pi^{*} \mathcal{D}^{ \pm} \cdot \pi^{*} \mathcal{D}^{ \pm}
$$

We have

$$
\pi^{*} \mathcal{D}^{ \pm}=\sum_{g \in G} g\left(D^{ \pm}\right)
$$

We get

$$
\begin{aligned}
\mathcal{D}^{ \pm} \cdot \mathcal{D}^{ \pm} \cdot \mathcal{D}^{ \pm}= & \frac{1}{16} \sum_{g_{1}, g_{2}, g_{3}} g_{1}\left(D^{ \pm}\right) \cdot g_{2}\left(D^{ \pm}\right) \cdot g_{3}\left(D^{ \pm}\right)= \\
& \sum_{g_{1}, g_{2}} D^{ \pm} \cdot g_{1}\left(D^{ \pm}\right) \cdot g_{2}\left(D^{ \pm}\right) .
\end{aligned}
$$

With the results of the previous section we can derive now our main result.

4.3 Theorem. The two divisors $\mathcal{D}^{ \pm}$of the rigid Calabi-Yau manifold $\tilde{\mathcal{Y}}:=$ $\tilde{\mathcal{X}} / G$ give a basis of $\operatorname{Pic} \tilde{\mathcal{Y}} \otimes_{\mathbb{Z}} \mathbb{Q}$. Their intersection numbers are

$$
\mathcal{D}^{+} \cdot \mathcal{D}^{+} \cdot \mathcal{D}^{+}=\mathcal{D}^{-} \cdot \mathcal{D}^{-} \cdot \mathcal{D}^{-}=2^{7}, \quad \mathcal{D}^{+} \cdot \mathcal{D}^{+} \cdot \mathcal{D}^{-}=\mathcal{D}^{+} \cdot \mathcal{D}^{-} \cdot \mathcal{D}^{-}=5 \cdot 2^{7}
$$


The intersection numbers get nicer if one uses a different basis of Pic $\mathcal{Y} \otimes_{\mathbb{Z}} \mathbb{Q}$, namely

$$
\mathcal{A}=\frac{1}{16}\left(\mathcal{D}^{+}+\mathcal{D}^{-}\right), \quad \mathcal{B}=\frac{1}{8}\left(\mathcal{D}^{+}-\mathcal{D}^{-}\right)
$$

Then we get

$$
\mathcal{A}^{3}=1, \quad \mathcal{B}^{3}=\mathcal{A}^{2} \mathcal{B}=0, \quad \mathcal{A B}^{2}=-1
$$

There may be several projective small resolutions $\tilde{\mathcal{X}}$ of $\mathcal{X}$ such that $G$ extends to it. The results show that the intersection numbers are in all cases the same.

\section{References}

[CM] Cynk, S., Meyer, C.: Modular Calabi-Yau Threefolds of level eight, Internat. J. Math. 18, no. 3, 331-347 (2007)

[FS] Freitag, E., Salvati-Manni, R.: On Siegel threefolds with a projective CalabiYau model, Commun. Number Theory Phys. 5, No. 3, 713-750 (2011)

[GN] van Geemen, B., Nygaard, N.O.: On the geometry and arithmetic of some Siegel modular threefolds, Journal of Number Theory 53, 45-87 (1995)

[Sh] Shafarevich, I.R.: Basic Algebraic Geometry, Springer-Verlag Berin Heidelberg New York (1974)

Eberhard Freitag

Mathematisches Institut

Im Neuenheimer Feld 288

D69120 Heidelberg

freitag@mathi.uni-heidelberg.de 